\title{
Exploring the use of a column model for the characterization of microphysical processes in warm rain: results from a homogeneous rainshaft model
}

\author{
O. P. Prat and A. P. Barros \\ Civil and Environmental Engineering Department, Pratt School of Engineering, Duke University, Durham NC 27708, USA
}

Received: 31 July 2006 - Revised: 29 November 2006 - Accepted: 27 January 2007 - Published: 26 April 2007

\begin{abstract}
A study of the evolution of raindrop spectra (raindrop size distribution, DSD) between cloud base and the ground surface was conducted using a column model of stochastic coalescense-breakup dynamics. Numerical results show that, under steady-state boundary conditions (i.e. constant rainfall rate and DSD at the top of the rainshaft), the equilibrium DSD is achieved only for high rain rates produced by midlevel or higher clouds and after long simulation times ( $\sim 30 \mathrm{~min}$ or greater). Because these conditions are not typical of most rainfall, the results suggest that the theoretical equilibrium DSD might not be attainable for the duration of individual rain events, and thus DSD observations from field experiments should be analyzed conditional on the specific storm environment under which they were obtained.
\end{abstract}

\section{Introduction}

The evolution of rainfall microstructure in the presence of combined coalescence, breakup, accretion, evaporation, and condensation mechanisms has been the object of extensive research over the past 40 years (Testik and Barros, 2007). The ability to simulate the rain drop size distribution (DSD) in the natural environment is key for studying precipitation processes generally, and to validate microphysical parameterizations in numerical models. In this work, a column model of the stochastic equation describing the evolution of raindrop spectra through a rainshaft has been developed. Previously, Prat and Barros (2007) tested several coalescence/breakup kernels for a single box model in which the spatial homogeneity of the DSD is assumed, and the evolution of the DSD is tracked over time. The single box model is extended in this study to a homogeneous onedimensional rainshaft model in which the evolution of the

Correspondence to: A. P. Barros

(barros@duke.edu)
DSD is observed as the drops fall through the atmospheric column (List et al., 1987; Tzivion et al., 1989; List and McFarquhar, 1990; Hu and Srivastava, 1995). The specific research goal is to establish a model that predicts the evolution of the rainfall microstructure for a simple configuration describing coalescence-breakup dynamics below cloud base. More broadly, this work is a first step toward a simulation of the evolution of rainfall microstructure in warm rain with detailed microphysics in complex weather prediction models, and to achieve a dynamic simulation of DSD that will be used for physical algorithms in radar rainfall estimation.

\section{Model description}

2.1 Discretization of the general Stochastic Collection/Stochastic Breakup Equation

A full description of the box-model is provided by Prat and Barros (2007). We present here only a summary of the key components for the rainshaft implementation. The general governing equation for the rate of change of the number concentration $n(v, z, t)$ of drops with volume between $v$ and $v+d v$ in a vertical rainshaft in the presence of coalescence and breakup is given by the following general equation:

$$
\begin{aligned}
& \frac{\partial n(v, z, t)}{\partial t}+\frac{\partial}{\partial z}[n(v, z, t) V(v, z)]=S(v, z, t) \\
& =\frac{1}{2} \int_{0}^{v} n\left(v-v^{\prime}, t\right) n\left(v^{\prime}, t\right) C\left(v-v^{\prime}, v^{\prime}\right) d v^{\prime} \\
& -n(v, t) \int_{0}^{\infty} n\left(v^{\prime}, t\right) C\left(v, v^{\prime}\right) d v^{\prime} \\
& +\frac{1}{2} \int_{0}^{\infty} n\left(v^{\prime}, t\right) d v^{\prime} \int_{0}^{\infty} n\left(v^{\prime \prime}, t\right) B\left(v^{\prime}, v^{\prime \prime}\right) P\left(v, v^{\prime}, v^{\prime \prime}\right) d v^{\prime \prime} \\
& -n(v, t) \int_{0}^{\infty} n\left(v^{\prime}, t\right) v^{\prime} B\left(v, v^{\prime}\right) d v^{\prime} \\
& =I_{1}(v, z, t)+I_{2}(v, z, t)+I_{3}(v, z, t)+I_{4}(v, z, t)
\end{aligned}
$$

The first term on the left hand side of (Eq. 1) represents the temporal evolution of the droplet number density $n(v, z, t)$ between volume (i.e. mass) interval $v$ and $v+d v$, at location

Published by Copernicus GmbH on behalf of the European Geosciences Union. 
$z$ and time $t$. The second term represents the fall of a drop at a velocity $V(v, z, t)$. The source term $S(v, z, t)$ on the right hand side describes the coalescence-breakup dynamics. The coalescence of droplets is described by terms $I_{1}$ (rate of creation of droplet of volume $v$ due to the coalescence of smaller drops), and $I_{2}$ (rate of disappearance of droplet of volume $v$ due to coalescence with other drops of volume $v^{\prime}$ ). The collisional breakup mechanism is described by terms $I_{3}$ (rate of creation of drop of volume $v$ resulting from a collisional breakup event involving two drops of volume $v^{\prime}$ and $v^{\prime \prime}$ ), and $I_{4}$ (rate of disappearance of drops of volume $v$ involved in a collisional breakup event with other drops). Terms $C\left(v, v^{\prime}\right)$, $B\left(v, v^{\prime}\right)$, and $P\left(v, v^{\prime}, v^{\prime \prime}\right)$ are respectively the collection kernel, the breakup kernel and the fragment distribution function by collisional breakup involving two drops of volume $v^{\prime}$ and $v^{\prime \prime}$ for a resulting droplet of volume $v$.

In the rainshaft model, we assume homogeneity with regard to the height $(z)$ for the fall velocity of drops of volume $v V(v, z)=V(v)$. Finally, the term describing the fall of drops through the atmospheric column is reduced to:

$$
\frac{\partial}{\partial z}[n(v, z, t) V(v, z)]=V(v) \frac{\partial}{\partial z}[n(v, z, t)]
$$

The source term is discretized using a fixed pivot technique (Kumar and Ramkrishna, 1996) that allows the discrete equation to be internally consistent with two selected integral properties of the DSD. More details concerning the intermediate step for the discretization of the source term in the general Stochastic Collection/Stochastic Breakup Equation (SC/SBE) can be found in Kumar and Ramkrishna (1996) for the coalescence part of the equation, and Prat and Barros (2007) for the extension to collisional breakup. Finally, the integration of Eq. (1) over the $i$ th discrete interval is given by (Prat and Barros, 2007) :

$$
\begin{aligned}
& \frac{d N_{i}(z, t)}{d t}+V_{i} \frac{d N_{i}(z, t)}{d z}=S_{i}(z, t) \\
& =\sum_{j, k}^{j \geq k}\left(1-\frac{1}{2} \delta_{j, k}\right) \eta C_{j, k} N_{j}(z, t) N_{k}(z, t) \\
& x_{i-1} \leq\left(x_{j}+x_{k}\right) \leq x_{i+1} \\
& -N_{i}(z, t) \sum_{k=1}^{\text {nbin }} C_{i, k} N_{k}(z, t) \\
& +\frac{1}{2} \sum_{j=1}^{\text {nbin nbin }} \sum_{k=1}^{n} N_{j}(z, t) N_{k}(z, t) B_{i, k} \kappa_{i, j, k} \\
& -N_{i}(z, t) \sum_{k=1}^{\text {nbin }} B_{i, k} N_{k}(z, t)
\end{aligned}
$$

Where $N_{i}(z, t)$ is the total number density of droplet in the $i$ th class size $\left(\mathrm{cm}^{-3}\right)$ :

$N_{i}(z, t)=\int_{v_{i}}^{v_{i+1}} n(v, z, t) d v$ and

$$
\begin{aligned}
& C_{i, j}=C\left(x_{i}, x_{j}\right)=K\left(x_{i}, x_{j}\right) \cdot E_{(\mathrm{coal})}\left(x_{i}, x_{j}\right)=K_{i, j} \cdot E_{(\mathrm{coal}) i, j} \\
& B_{i, j}=B\left(x_{i}, x_{j}\right)=K\left(x_{i}, x_{j}\right) \cdot E_{(\mathrm{brkp})}\left(x_{i}, x_{j}\right)=K_{i, j} \cdot\left(1-E_{(\mathrm{coal}) i, j}\right)
\end{aligned}
$$

where $E_{\text {(coal) }}\left(x_{i}, x_{j}\right)$ is the coalescence efficiency and $K\left(x_{i}, x_{j}\right)$ is the gravitational collection kernel:

$$
K_{i, j}=[9 \pi / 16]^{1 / 3} \cdot\left(x_{i}^{1 / 3}+x_{j}^{1 / 3}\right)^{2}\left|V_{i}-V_{j}\right|
$$

The terms $\eta$ and $\kappa_{i, j, k}$ are derived from the selected discretization method. The term $\eta$ can be seen as the contribution to droplet population located at the $i$ th size interval due to the coalescence of two droplets of size $x_{j}$ and $x_{k}$, respectively, and the term $\kappa_{i, j, k}$ can be seen as the contribution to the droplet population located at the $i$ th size interval due to the collisional breakup of two droplets of size $x_{j}$ and $x_{k}$, respectively. For the selected discretization scheme internally consistent with respect to the drop number density $\left(M_{0}\right)$ and drop mass concentration $\left(M_{1}\right), \eta$ is equal to :

$\eta=\frac{x_{i+1}-v}{x_{i+1}-x_{i}} \quad$ if $\quad x_{i} \leq v \leq x_{i+1}$

and $\quad \eta=\frac{v-x_{i-1}}{x_{i}-x_{i-1}} \quad$ if $\quad x_{i-1} \leq v \leq x_{i}$

and $\kappa_{i, j, k}$ is given by :

$$
\begin{aligned}
\kappa_{i, j, k} & =\int_{x_{i}}^{x_{i+1}} \frac{x_{i+1}-v}{x_{i+1}-x_{i}} P\left(v, x_{j}, x_{k}\right) d v \\
& +\int_{x_{i-1}}^{x_{i}} \frac{v-x_{i-1}}{x_{i}-x_{i-1}} P\left(v, x_{j}, x_{k}\right) d v
\end{aligned}
$$

Finally, the average number density of droplets for the $i$ th size category (between diameter $d_{i}$ and $d_{i+1}$ in $\mathrm{cm}$ ) by size interval $\left[\mathrm{cm}^{-3} \mathrm{~cm}^{-1}\right]$ is given by :

$\bar{n}_{i}(z, t)=\frac{1}{d_{i+1}-d_{i}} \int_{v_{i}}^{v_{i+1}} n(v, z, t) d v=\frac{N_{i}(z, t)}{d_{i+1}-d_{i}}$

Thus, the $k$ th order moment of the DSD is :

$M_{k}(z, t)=\sum_{i=1}^{\text {nbin }} N_{i}(z, t) m_{i}^{k}$

where $m_{i}$ is the drop mass (in [g]) in the $i$ th class category. Moments of interest include the drop number concentration $\left(M_{k=0}\right.$ in $\left.\left[\mathrm{cm}^{-3}\right]\right)$, the drop mass concentration or liquid water content $\left(M_{k=1}\right.$ in $\left.\left[\mathrm{g} \mathrm{cm}^{-3}\right]\right)$, and radar reflectivity factor $\left(Z=[6 /(\rho \cdot \pi)]^{2} \cdot 10^{12} \cdot M_{k=2}\right.$ in $\left.\left[\mathrm{mm}^{6} \mathrm{~m}^{-3}\right]\right)$. Equation (3) is then solved using a simple forward finite difference scheme and transformed into:

$$
\begin{aligned}
N_{i}\left(z_{h}, t+\Delta t\right) & =N_{i}\left(z_{h}, t\right)+V_{i} \cdot \frac{\Delta t}{\Delta z} \cdot\left[N_{i}\left(z_{h-1}, t\right)-N_{i}\left(z_{h}, t\right)\right] \\
& +S_{i}\left(z_{h}, t\right) . \Delta t
\end{aligned}
$$


(a)

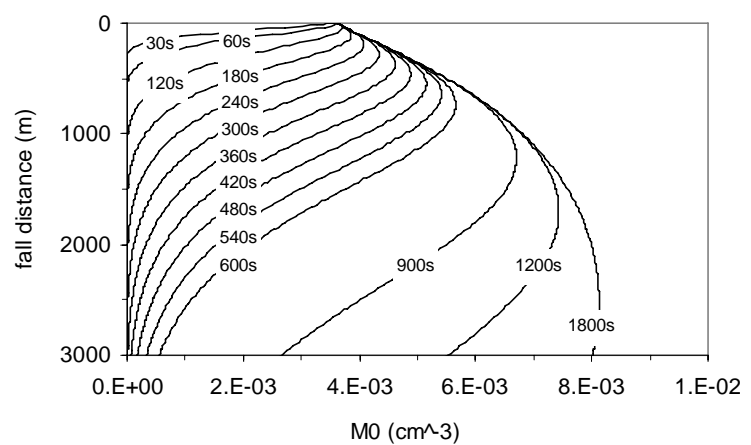

(b)

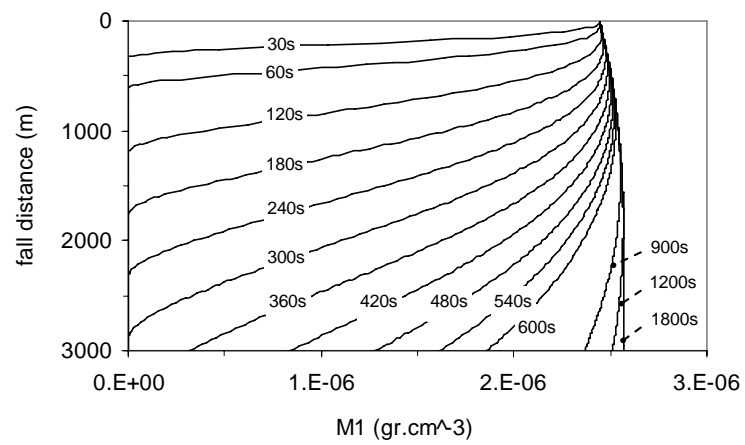

(c)

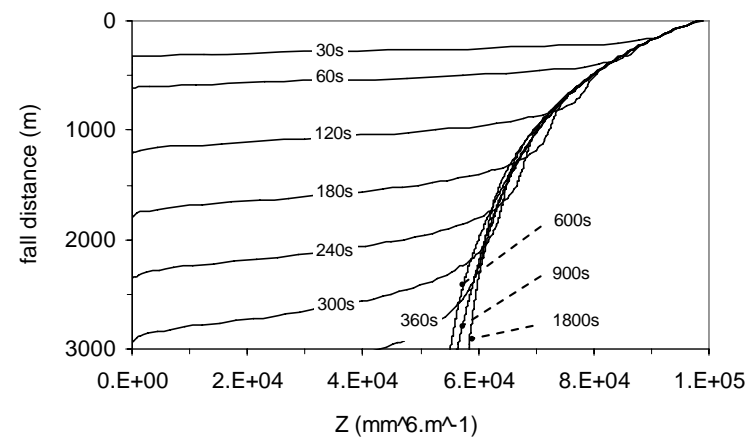

Fig. 1. Evolution of vertical profiles of DSD integral properties for the time dependent column model: (a) Droplet number density $\left(M_{0}\right)$. (b) Droplet mass $\left(M_{1}\right)$. (c) Radar reflectivity factor $\left(Z=[6 /(\rho . \pi)]^{2} \cdot M_{2}\right.$. The initial DSD is a Marshall-Palmer distribution with a nominal rainrate $R=50 \mathrm{~mm} \cdot \mathrm{h}^{-1}$.

Numerical stability of (12) is achieved for $1>V_{i} . \Delta t / \Delta z$ where $(\Delta z)$ is the spatial resolution and $(\Delta t)$ is the time step. The expression for the fall velocity $\left(V_{i}\right)$ was taken from Best (1950) with the largest drops of the DSD $(d=0.7 \mathrm{~cm})$ having a maximum fall velocity of about $9.5 \mathrm{~m} \cdot \mathrm{s}^{-1}$.

\subsection{Formulation of coalescence-breakup kinetics for the $\mathrm{SC} / \mathrm{SBE}$}

The discrete model for the resolution of the SC/SBE is used for the prediction of the evolution of the DSD in the presence of coalescence and collisional breakup. The expression for the coalescence efficiency is from by Low and List

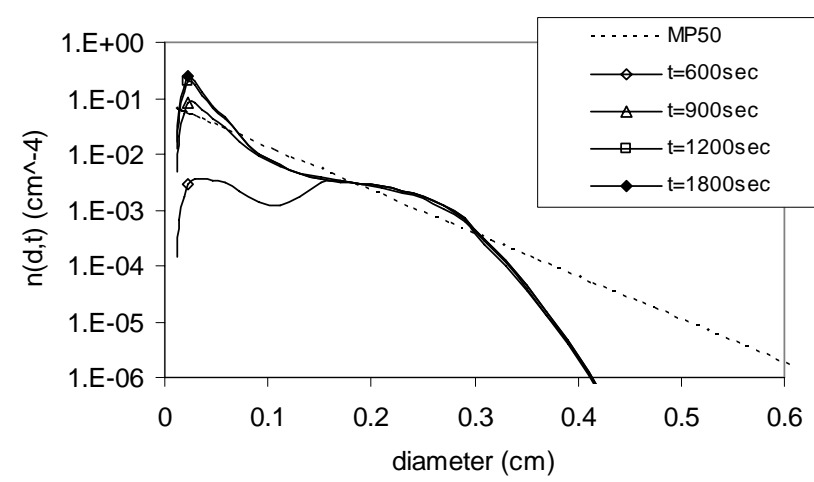

Fig. 2. DSD evolution of the solution of the SC/SBE for the time dependent column model after a fall distance of $3000 \mathrm{~m}$. The initial DSD is a Marshall-Palmer distribution with a nominal rainrate $R=50 \mathrm{~mm} \cdot \mathrm{h}^{-1}$. The solution is obtained for an irregular grid (40 bins).

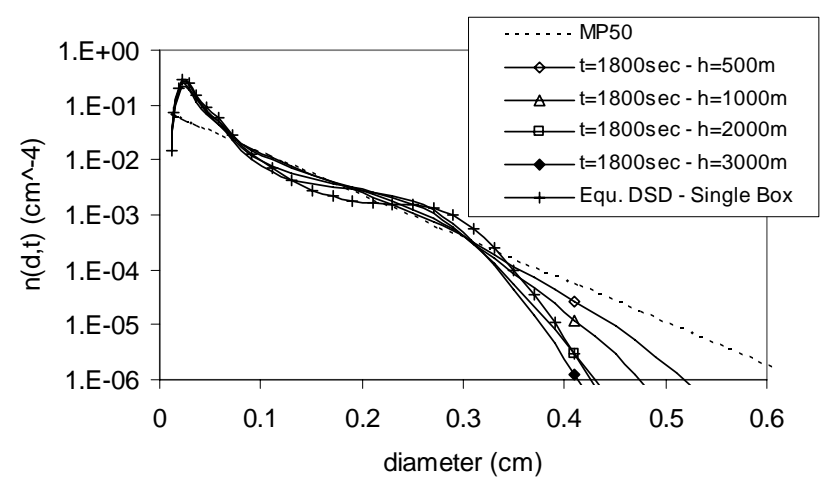

Fig. 3. DSD at $t=1800 \mathrm{~s}$ for the solution of the SC/SBE for the time dependent column model for different fall height values. The initial DSD is a Marshall-Palmer distribution with a nominal rainrate $R=50 \mathrm{~mm} \cdot \mathrm{h}^{-1}$. The solution is obtained for an irregular grid (40 bins). The Equilibrium DSD obtained with the homogenous in space, transient in time, single box model is reported for comparison.

(1982a). McTaggart-Cowan and List (1975) and later Low and List (1982a, b) performed collision experiments with two drops of different diameter and identified three types of breakup (disc: DI, filament: FI, and sheet: SH). Low and List (1982a,b) proposed expressions of the ratio for each type of breakup ( $R_{\mathrm{DI}}$ : disc, $R_{\mathrm{FI}}$ : filament, and $R_{\mathrm{SH}}$ : sheet) and for the number of fragments created $\left(F_{\mathrm{DI}}\right.$ : disc, $F_{\mathrm{FI}}$ : filament, and $F_{\mathrm{SH}}$ : sheet). In addition, Low and List (1982a, b) found that the overall fragment distribution function for each pair of colliding drops is a sum of 2(3) lognormal and Gaussian distributions depending on the type of breakup (DI(2), $\mathrm{FI}(3), \mathrm{SH}(2))$. A complete description of the fragment distribution function requires the determination of parameters of the lognormal/Gaussian distributions such as the height $(\mathrm{H})$, the standard deviation $(\sigma)$, and the modal diameter $(\mu)$ 
(a)

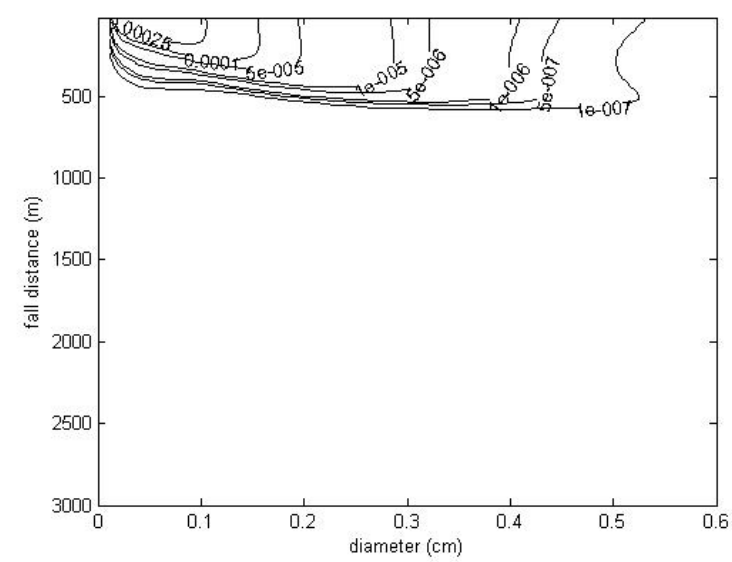

(c)

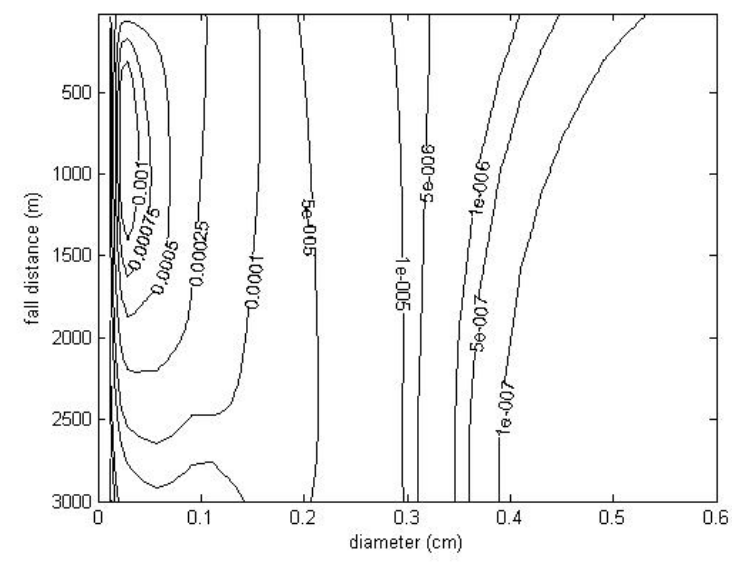

(b)

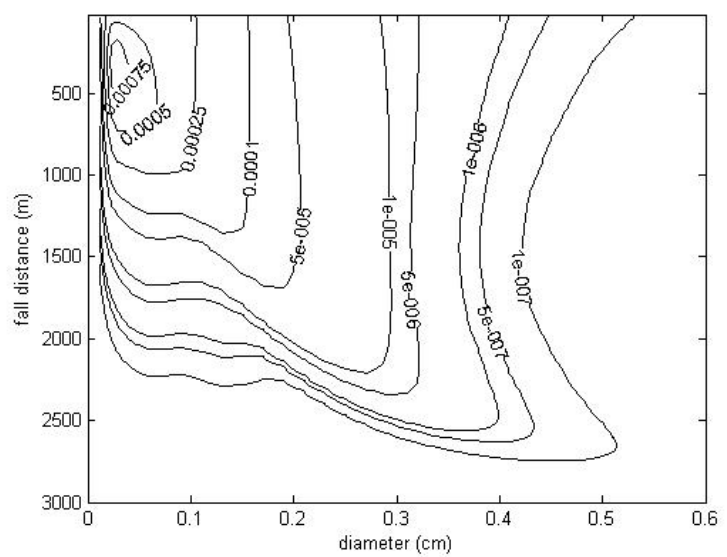

(d)

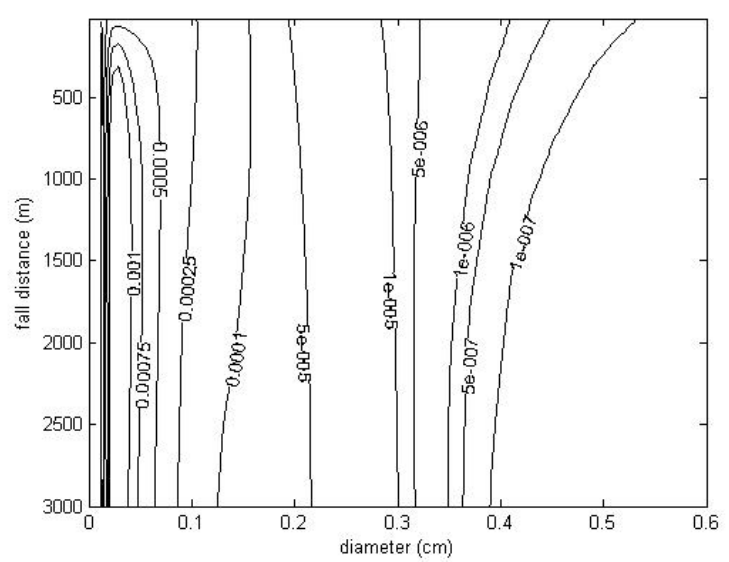

Fig. 4. Number distribution (in $\left[\mathrm{cm}^{-3}\right]$ ) for the time dependent column model (a) $t=60 \mathrm{~s}$. (b) $t=300 \mathrm{~s}$. (c) $t=600 \mathrm{~s}$. (d) $t=1800 \mathrm{~s}$. The initial DSD is a Marshall-Palmer distribution with a nominal rainrate $R=50 \mathrm{~mm} \cdot \mathrm{h}^{-1}$. The solution is obtained for an irregular grid (40 bins).

of the distribution for each pair of colliding drops. We use the formulation proposed by McFarquhar (2004), which was derived from Low and List (1982a, b) experimental data for the fragment distribution function. McFarquhar (2004) used a modified Monte Carlo method with bootstrap to randomly choose the result of the collision of arbitrary pairs of drops and proposed general expressions for the parameters $(H, \sigma$, $\mu$ ) of the fragment distribution functions for each type of breakup. This parameterization has the double advantage of having a more consistent physical basis in order to generalize experimental results of collisional breakup to arbitrarily selected pairs of colliding drops, thus overcoming the challenge posed by the original Low and List parameterization $(1982 \mathrm{a}, \mathrm{b})$ in terms of mass conservation enforcement for each breakup event. The latter aspect has been a major challenge concerning the implementation of the Low and List parameterization in drop coalescence/breakup models (Valdez and Young, 1985; Brown, 1987, 1988, 1997). A more complete description of the numerical model as well as a detailed discussion concerning mass conservation considerations for each single breakup event can be found in Prat and Barros (2007).

\section{Modeling results for a homogeneous one-dimensional rainshaft model}

As pointed out earlier, the fixed pivot method (Kumar and Ramkrishna, 1996) used in this work allows the selection of any type of discretization grid. A grid sensitivity analysis was performed in order to determine a good compromise between computational efficiency and accuracy of the DSD. Typically a geometric grid with a parameter $s=2^{1 / 3}$ can describe accurately the DSD as reported in previous studies ( $\mathrm{Hu}$ and Srivastava, 1995; Prat and Barros, 2007). We use here an irregular grid (40 bins) that covers a diameter range from $0.01 \mathrm{~cm}$ to $0.7 \mathrm{~cm}$. The selected grid combines a geometric grid $(s=2)$ at small drop size $(d \leq 0.1 \mathrm{~cm})$ in order to capture 
(a)

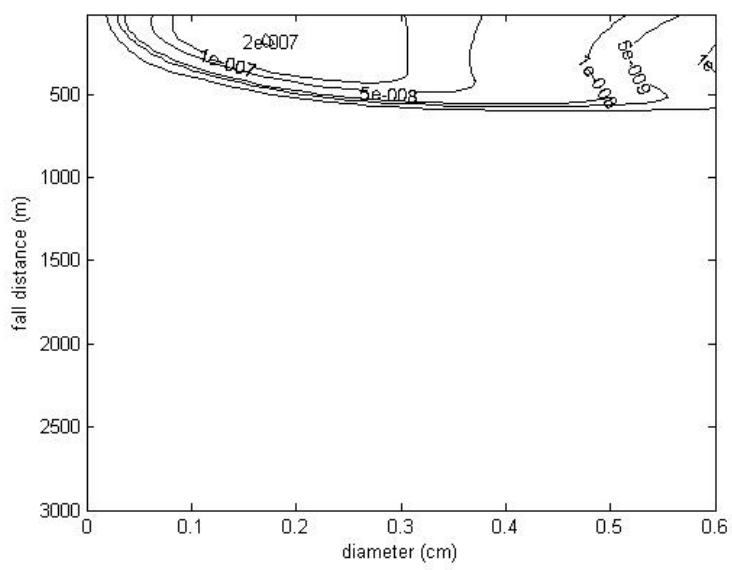

(c)

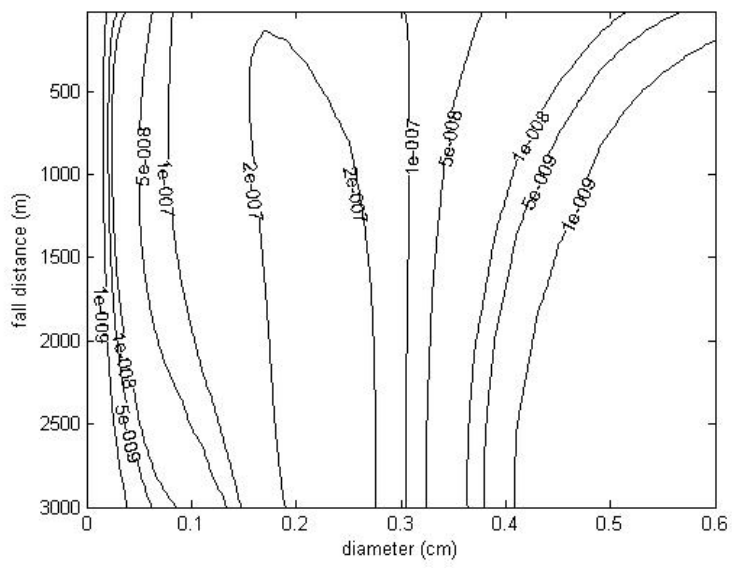

(b)

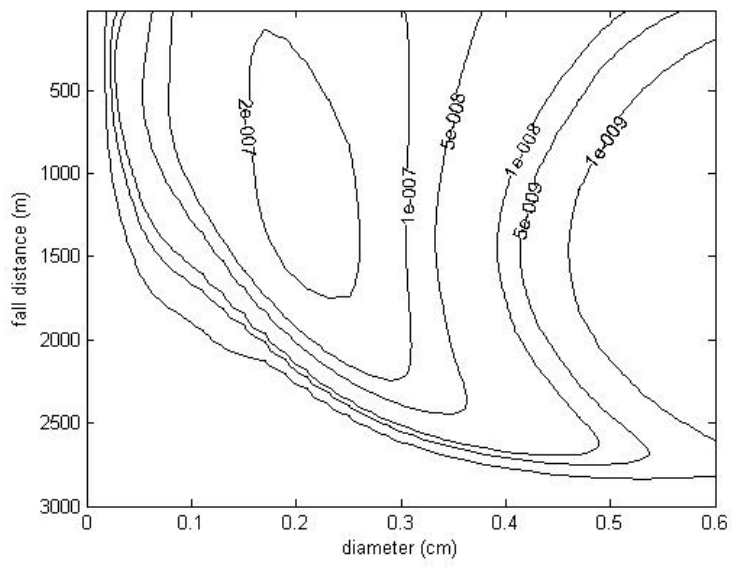

(d)

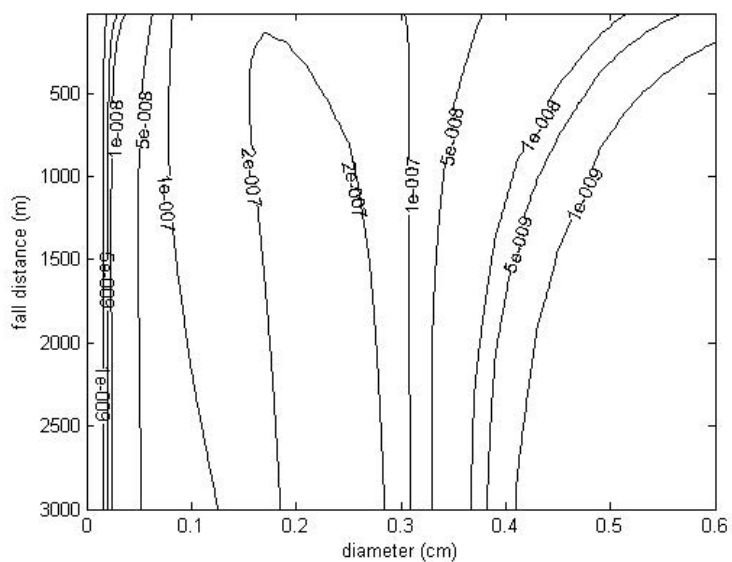

Fig. 5. Mass distribution or Liquid Water Content (in $\left[\mathrm{gr} \mathrm{cm}^{-3}\right]$ ) for the time dependent column model (a) $t=60 \mathrm{~s}$. (b) $t=300 \mathrm{~s}$. (c) $t=600 \mathrm{~s}$. (d) $t=1800 \mathrm{~s}$. The initial DSD is a Marshall-Palmer distribution with a nominal rainrate $R=50 \mathrm{~mm} \cdot \mathrm{h}^{-1}$. The solution is obtained for an irregular grid (40 bins).

accurately the location of peaks in the small drop diameter range, and a regular grid $(\Delta d=0.02 \mathrm{~cm})$ at larger drop sizes $(d \geq 0.1 \mathrm{~cm})$ in order to capture accurately the tail of the DSD and minimize the numerical diffusivity. Compared to a fine geometric grid $\left(s=2^{1 / 3}, 60\right.$ bins), the description of the tail is improved at a lower computational cost. The results presented in this study were obtained for a $3000 \mathrm{~m}$ height rainshaft with a vertical resolution $(\Delta z=20 \mathrm{~m})$ and a time step $(\Delta t=2 \mathrm{~s})$.

Figure 1 displays the temporal evolution of the vertical profiles of DSD integral properties, i.e. drop number concentration $\left(M_{0}\right.$ in $\left.\left[\mathrm{cm}^{-3}\right]\right)$, liquid water content $\left(\mathrm{LWC}=M_{1}\right.$ in $\left.\left[\mathrm{gr} \mathrm{cm}^{-3}\right]\right)$, and radar reflectivity $\left(Z=[6 /(\rho . \pi)]^{2} \cdot 10^{12} \cdot M_{2}\right.$ in $\left[\mathrm{mm}^{6} \cdot \mathrm{m}^{-1}\right]$ ) for an initial Marshall-Palmer (MP) distribution at the top of the rainshaft with a nominal rainrate of $50 \mathrm{~mm} . \mathrm{h}^{-1}$ comparable with values commonly used in similar studies $\left(50 \mathrm{~mm}^{-1} \mathrm{~h}^{-1}\right.$ : List et al., 1987; List and McFar- quhar, 1990; $100 \mathrm{~mm} \cdot \mathrm{h}^{-1}$ : Tzivion et al., 1989; Hu and Srivastava, 1995). The results show the evolution of the raindrop spectra below cloud basis as drops are falling through the air and reach the ground in the absence of updraft. Evaporation/condensation processes below cloud basis are ignored. In Fig. 1a, the drop number density is found to increase with increasing drop fall distance due to the fact that more collisional breakup events occur with increasing fall distance and that more smaller drops are created. In other words, number density increases between cloud base and the ground. The vertical mass profile remains almost constant, with a relative variation of less than $2 \%$, along the rainshaft (Fig. 1b). Due to the fact that the radar reflectivity factor $(Z)$ is proportional to the second order moment $\left(M_{2}\right), Z$ is found to decrease with increasing fall distance because of the collisional breakup of larger drops, causing reflectivity to decrease from cloud top to cloud base. In addition, the evolution toward a 
(a)

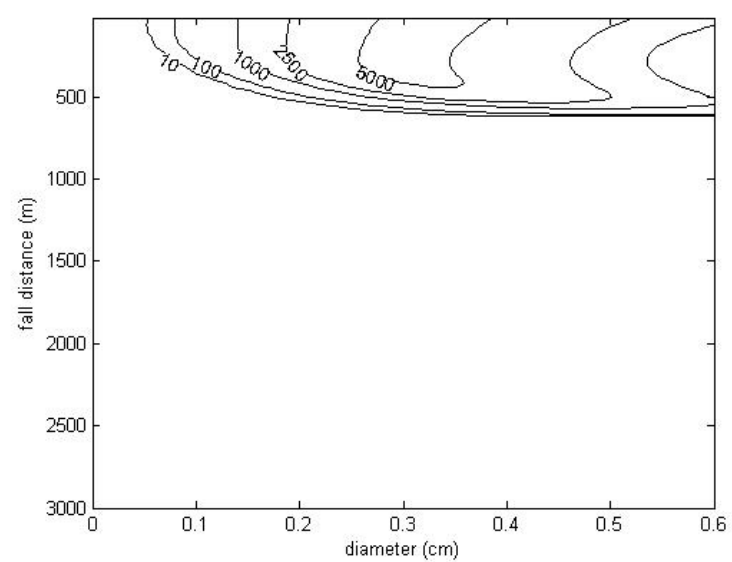

(c)

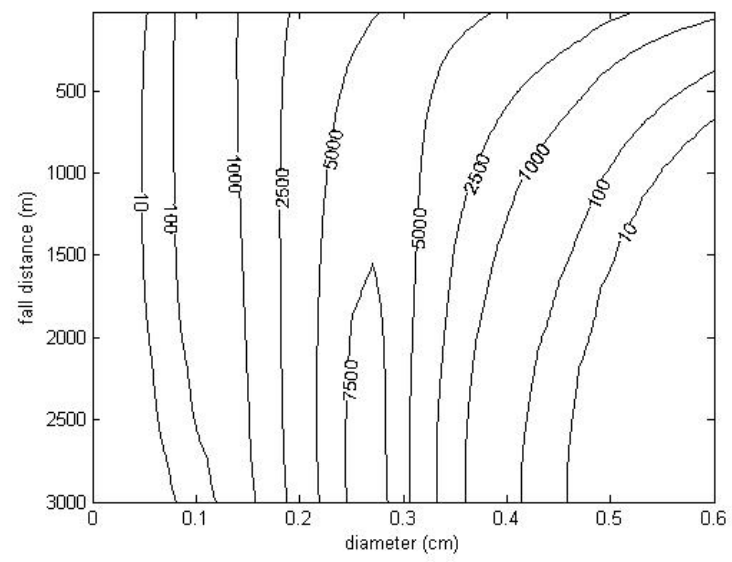

(b)

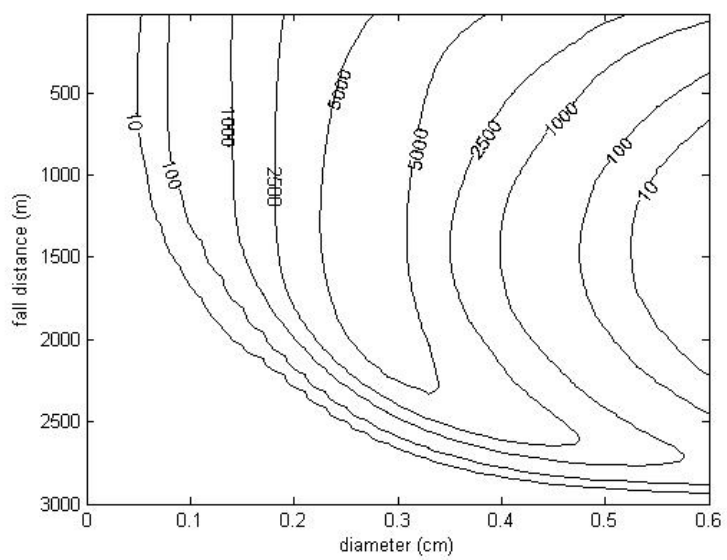

(d)

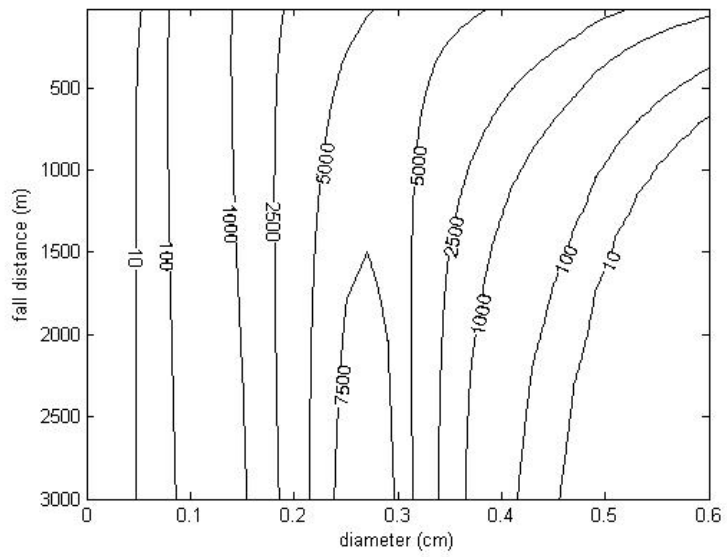

Fig. 6. Radar reflectivity factor $Z$ (in $\left[\mathrm{mm}^{6} \mathrm{~m}^{-1}\right]$ ) for the time dependent column model (a) $t=60 \mathrm{~s}$. (b) $t=300 \mathrm{~s}$. (c) $t=600 \mathrm{~s}$. (d) $t=1800 \mathrm{~s}$. The initial DSD is a Marshall-Palmer distribution with a nominal rainrate $R=50 \mathrm{~mm} \cdot \mathrm{h}^{-1}$. The solution is obtained for an irregular grid (40 bins).

steady state situation is faster for higher order moments as can be seen when comparing profiles of the droplet number density $\left(M_{0}\right.$ : Fig. $\left.1 \mathrm{a}\right)$ and the radar reflectivity factor $(Z$ : Fig. 1c) at times $600 \mathrm{~s}$ and $3600 \mathrm{~s}$.

Figure 2 displays the evolution of the DSD after a $3000 \mathrm{~m}$ fall through the rainshaft with a continuous Marshall-Palmer distribution with a nominal rainrate of $50 \mathrm{~mm} \cdot \mathrm{h}^{-1}$ imposed at the top. For the time interval sufficient for drops to reach the observation altitude $(t=600 \mathrm{~s})$, the DSD contains a higher number of large drops. However, due to the collisional breakup processes involving large drops, the peak of the DSD evolves towards smaller drop diameters over time. The number of small drops increases with increasing simulation time, causing the DSD to evolve towards a single peak curve with the peak located at $d=0.026 \mathrm{~cm}$ as observed in the case of the single model (Prat and Barros, 2007).
Figure 3 shows the profile of the steady state DSD achieved for different fall distances. The number of large drops decreases with increasing fall distance. The DSD presents a steeper tail with increasing fall distance due to the fact that breakup contribution for larger drops increases with increasing fall distance. As the fall distance increases, the tail of the DSD tends towards a steeper MP distribution. In addition, the height of the peak located at $0.026 \mathrm{~cm}$ increases with increasing fall distance due to the creation of smaller drops by breakup. Results for the column model are compared with the equilibrium DSD obtained with the single box model (Prat and Barros, 2007) for the same coalescence (Low and List, 1982a) and breakup (MF04) kernels. It is interesting to notice that compared to the single box model results (Prat and Barros, 2007), the DSD from the rainshaft model does not show the second peak located at $d=0.25 \mathrm{~cm}$, but only a shoulder near the same diameter range. Differences between 
equilibrium DSDs obtained with the rainshaft model for various fall distances and with the single box are explained by the fact that a true equilibrium DSD is not reached for the fall distances considered (up to $3000 \mathrm{~m}$ ) and that would be eventually achieved for higher fall distances. That is, the rainshaft does not reach steady state. In the case of the box model, the DSD at the previous time step is set as initial condition to compute the next time step DSD and the equilibrium solution is achieved faster than in the case of the column model where the same MP50 DSD is constantly introduced at the top of the rainshaft.

Figures 4, 5, and 6 display, respectively, the vertical profiles of raindrop number concentration $\left(N_{i}(z, t)\right)$, raindrop mass concentration $\left(N_{i}(z, t) . x_{i}\right.$ : with $x_{i}$ being the mass characteristic of the $i$ th size class), and radar reflectivity factor $\left([6 /(\rho \cdot \pi)]^{2} \cdot N_{i}(z, t) \cdot 10 e^{12} \cdot x_{i}^{2}\right)$. At the beginning of the simulation, we observe that larger drops tend to fall without significant breakup (see the $\mathrm{C}$ shaped profiles at $t=60 \mathrm{~s}$ (Figs. $4 \mathrm{a}$ and 5a), $t=300 \mathrm{~s}$ (Figs. $4 \mathrm{~b}$ and 5b), and $t=600 \mathrm{~s}$ (Figs. $4 \mathrm{c}$ and 5c). As time evolves, each level of the rainshaft (i.e. cloud layer) is filled more homogeneously with all droplet sizes due to continuous settling and the creation of smaller drops by breakup. The collisional breakup mechanism becomes enhanced and with increasing simulation time, a steady state vertical profile is achieved (Figs. $4 d$ and 5d). Figure $4 d$ shows that the maximum for the number distribution is located in the domain of smaller drop diameters in the vicinity of a diameter $d=0.026 \mathrm{~cm}$. For the mass profiles (Fig. $5 \mathrm{~d}$ ), the maximum is observed at drop diameters between $0.2 \mathrm{~cm}$ and $0.3 \mathrm{~cm}$, which corresponds to the location of the shoulder of the DSD. A similar behavior is observed for the radar reflectivity factor $(Z)$ with maximum located around drop diameter $0.25 \mathrm{~cm}$.

\section{Conclusions}

A homogeneous one-dimensional rainshaft model has been developed to investigate the dynamics of coalescence/collisional breakup. This model uses a fixed pivot technique that insures the conservation of two selected properties of the DSD. This model was previously tested for some coalescence/breakup kernels found in the literature in the case of a box configuration (Prat and Barros, 2007). In the present study, results were presented for a combined coalescence-breakup mechanism in the case of a homogeneous rainshaft model. The model uses the parameterization proposed by McFarquhar (2004). Numerical results have shown that an equilibrium DSD is achieved only for significant fall distances (for cloud basis located $3000 \mathrm{~m}$ above ground level) and long simulation times ( $30 \mathrm{~min})$ for high rainrates $\left(50 \mathrm{~mm} \mathrm{~h}^{-1}\right)$. Those results suggest that for lighter rain events the establishment of an equilibrium situation might not be attainable for the duration of the rain event. For higher order moments of the DSD such as the liquid water content and the radar reflectivity factor, the establishment of equilibrium is achieved for shorter time periods.

The one-dimensional model is currently extended to more complex atmospheric configurations including updraft effects and evaporation-condensation processes in order to simulate microphysical processes occurring inside the cloud. In addition, modeling results are compared with experimental data including tower-based laboratory experiments with a focus on coalescence-breakup dynamics, and data collected during field campaigns using vertically pointing radar.

Acknowledgements. This research was supported in part by grants NSF ATM 97-530093 and NASA NNG04GP02G with the second author.

Edited by: S. C. Michaelides and E. Amitai

Reviewed by: anonymous referees

\section{References}

Best, A. C.: Empirical formulae for the terminal velocity of water drops falling through the atmosphere, Quart. J. Roy. Meteorol. Soc., 76, 302-311, 1950.

Brown, P. S.: Parameterization of drop-spectrum evolution due to coalescence and breakup, J. Atmos. Sci., 44(1), 242-249, 1987.

Brown, P. S.: The effects of filament, sheet, and disk breakup upon the drop spectrum, J. Atmos. Sci., 45(4), 712-718, 1988.

Brown, P. S.: Mass conservation considerations in analytic representation of raindrop fragment distributions, J. Atmos. Sci., 54, 1675-1687, 1997.

Hu, Z. and Srivastava, R. C.: Evolution of raindrop size distribution by coalescence, breakup, and evaporation: Theory and observations, J. Atmos. Sci., 52(10), 1761-1783, 1995.

Kumar, S. and Ramkrishna, D.: On the solution of population balance equations by discretization - I. A fixed pivot technique, Chem. Eng. Sci., 51(8), 1311-1332, 1996.

List, R., Donaldson, N. R., and Stewart, R. E.: Temporal evolution of drop spectra to collisional equilibrium in steady and pulsating rain, J. Atmos. Sci., 44(2), 362-372, 1987.

List, R. and McFarquhar, G. M.: The evolution of three-peak raindrop size distributions in one-dimensional shaft models. Part I: single-pulse rain, J. Atmos. Sci., 47(24), 2996-3006, 1990.

Low, T. B. and List, R.: Collision, coalescence and breakup of raindrops. Part I: Experimentally established coalescence efficiencies and fragment size distributions in breakup, J. Atmos. Sci., 39, 1591-1606, 1982a.

Low, T. B. and List, R.: Collision, coalescence and breakup of raindrops. Part II: Parameterization of fragment size distributions, J. Atmos. Sci., 39, 1607-1618, 1982 b.

McFarquhar, G. M.: A new representation of collision-induced breakup of raindrops and its implications for the shapes of raindrop size distributions, J. Atmos. Sci., 61(7), 777-794, 2004.

McTaggart-Cowan, J. D., and List, R.: Collision and breakup of water drops at terminal velocity, J. Atmos. Sci., 32, 1401-1411, 1975.

Prat, O. P. and Barros, A. P.: A robust numerical solution of the stochastic collection-breakup equation for warm rain, J. Appl. Meteorol. Climatol., in press, 2007. 
Testik, F. Y. and Barros, A. P.: Towards elucidating the microstructure of warm rainfall: A survey, Rev. Geophys, in press, 2007.

Tzivion, S., Feingold, G., and Levin, Z.: The evolution of raindrop spectra. Part II: Collisional collection/breakup and evaporation in a rainshaft, J. Atmos. Sci., 46(21), 3312-3327, 1989.
Valdez, M. P. and Young, K. C.: Number fluxes in equilibrium raindrop populations: A Markov chain analysis, J. Atmos. Sci., 42(10), 1024-1036, 1985. 